\title{
POTENCIAL DE MINERALIZAÇÃO DE N E DE C EM VINTE SOLOS DE PERNAMBUCO(1)
}

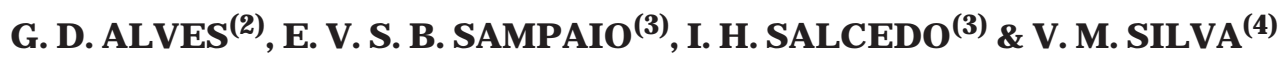

\begin{abstract}
RESUMO
A capacidade de mineralização de $\mathrm{C}$ e de $\mathbf{N}$ de 20 solos de Pernambuco (10 da zona úmida e 10 da semi-árida) foi determinada em casa de vegetação, entre janeiro e outubro de 1987, incubando-se amostras de $50 \mathrm{~g}$, das profundidades de 0-20 e 20-40 cm, e medindo-se, periodicamente, durante 20 e 4 semanas, respectivamente, o C-CO $\mathrm{CO}_{2}$ liberado e o $\mathrm{N}$ mineral $\left(\mathrm{NO}_{3}{ }^{-}\right.$e $\left.\mathrm{NH}_{4}{ }^{+}\right)$acumulado. As quantidades mineralizadas variaram muito entre os solos, mas, apesar disto, foram maiores na zona úmida que na semi-árida e, na semi-árida, foram maiores na camada de 0-20 que na de $20-40 \mathrm{~cm}$. Os solos apresentaram distintos padrões de mineralização ao longo da incubação, com a fase inicial com retardo ou com grande mineralização e a final com estabilização ou queda progressiva. As curvas de $\mathrm{C}$ tenderam mais à estabilização final que as de $\mathbf{N}$. As quantidades de $\mathrm{C}$ e de $\mathbf{N}$ mineralizados foram bem relacionadas entre si e com os teores de $\mathrm{C}$ e $\mathrm{N}$ totais do solo e as de $\mathbf{N}$ mineralizado com as quantidades absorvidas por plantas cultivadas em potes com os mesmos solos. A matéria orgânica da zona úmida tendeu a ser mais lábil que a da semi-árida, principalmente quanto ao $\mathrm{N}$, e a da camada superficial mais que a da subsuperficial.
\end{abstract}

Termos de indexação: $\mathrm{C}_{-} \mathrm{CO}_{2}$, matéria orgânica, zona tropical úmida, zona tropical semi-árida, nitrato, amônio

(1) Parte da Tese de Mestrado do primeiro autor, apresentada a Universidade F ederal Rural de Pernambuco, em abril/1989. Recebido para publicação em outubro de 1997 e aprovado em novembro de 1998.

(2) Biólogo, Departamento de Botânica, Universidade Federal de Pernambuco - UFPE. Av. Prof. Nelson Chaves s/n. CEP 50372-970 Recife (PE).

(3) Professor, Departamento de Energia Nuclear, UFPE. Av. Prof. Luiz Freire 1000, CEP 50740-540 Recife (PE). Bolsista do CNPq.

(4) Química, Departamento de Energia Nuclear, UFPE. 


\title{
SUMMARY: MINERALIZATION POTENTIAL OF C AND N IN TWENTY SOILS FROM PERNAMBUCO, BRAZIL
}

\begin{abstract}
The $\mathrm{C}$ and $\mathrm{N}$ mineralization potential of 20 soils from Pernambuco, Brazil (10 from the humid area and 10 from the semi-arid) was determined in a greenhouse experiment, conducted from J anuary to October 1987. Samples of $50 \mathrm{~g}$ from 0-20 and 20-40 cm depth layers wereincubated. Thereleased $\mathrm{C}-\mathrm{CO}_{2}$ and theaccumulated mi neral $\mathrm{N}\left(\mathrm{NO}_{3}{ }^{-}\right.$and $\left.\mathrm{NH}_{4}{ }^{+}\right)$ were periodically measured during 20 and 4 weeks, respectively. The mineralized amounts varied much among soils. In spite of this they were higher in the humid than in the semiarid area, being al sohigher in the 0-20 cm layer than in the 20-40 cm one. Thesoils presented different mineralization patterns al ong theincubation period: in thefirst weeks, there was either a lag or high mineralization and, in thelast weeks, therewas stabilization or progressive decline The $C$ curves tended to stabilization in the final phase than $\mathrm{N}$ curves. Mineralized amounts of $\mathrm{C}$ and $\mathrm{N}$ weresignificantly related to oneanother and to total soil $\mathrm{C}$ and $\mathrm{N}$ and mineralized $\mathrm{N}$ was related to $\mathrm{N}$ uptake by plants growing in pots with similar soils. Soil organic matter in the humid area was morelabilethan that in thesemi-arid area, especially regarding $\mathrm{N}$, and in the superficial layer rather than in the subsuperficial one.
\end{abstract}

Index terms: $\mathrm{C}-\mathrm{CO}_{2}$, organic matter, tropical humid area, tropical semi-arid area, nitrate, ammonium.

\section{NTRODUÇÃO}

A disponibilidade de nitrogênio do solo é, freqüentemente, limitante ao crescimento das plantas eà produtividade das culturas (Döbereiner, 1990). Por isso, el evadas quantidades de fertilizantes nitrogenados são adicionadas, anualmente, aos sol os. Apesar dessas adições, o $\mathrm{N}$ originado da mineralização da matéria orgânica do solo é considerado a fonte mais importante de $\mathrm{N}$ para as culturas, nas condições brasileiras (Malavolta, 1990). Tal importância justificaria grande esforço de pesquisa para determinar a capacidade de mineralização de $\mathrm{N}$ dos sol os brasileiros. Entretanto, são poucos os estudos nesta área (Kinjo et al., 1978; Pottker \& Tedesco, 1979; Sal cedo et al., 1985; Lemos et al., 1988; Parentoni et al., 1988; Camargo et al., 1997). U ma das razões da limitação destes estudos é a complexidade da dinâmica de $\mathrm{N}$ no sistema solo - planta que advém do grande número de fatores envolvidos. É também sabido que a dinâmica do $\mathrm{N}$ está muito relacionada com a do C, mas são praticamente inexistentes os trabal hos comparando as mineralizações simultâneas do $\mathrm{C}$ e do $\mathrm{N}$ da matéria orgânica dos sol os (F reytag \& Rausch, 1984; Salcedo et al., 1985).

Para estudar al guns desses fatores, tendo outros sob controle, foi montada uma série de experimentos em casa de vegetação e laboratório, com 20 sol os de Pernambuco (Sampaio et al., 1995). O objetivo específico destetrabal ho foi determinar a capacidade de mineralização de $\mathrm{C}$ e de $\mathrm{N}$ destes 20 solos, relacioná-la com seus teores de $\mathrm{C}$ e $\mathrm{N}$ totais e compará-la com a capacidade defornecimento deN, determinada anteriormente (Sampaio et al., 1995).

\section{MATERIAL E MÉTODOS}

Foram coletadas amostras de 20 solos em Pernambuco, 10 na zona úmida e 10 na zona semiárida, nas profundidades de 0-20 e 20-40 cm. Da zona úmida, os solos 1 a 4 foram classificados como Latossolos Vermel ho-Amarel os distróficos; o solo 5 como Terra Roxa Estruturada; os sol os 6 a 9 como Podzólicos Vermel ho-Amarel os Latossól icos distrófico; e o solo 10 como Glei Pouco Húmico distrófico. Da zona semi-árida, os sol os 11 a 13 foram classificados como Aluviais eutróficos, os solos 14 e 15 como Brunos Não-Cálcicos; os solos 16 e 17 como Regossólicos eutróficos; o solo 18 como Podzólico Vermelho-Amarelo eutrófico latossólico; o solo 19 como Planossol o Solódico; e o sol o 20 como Vertissolo Solódico. As amostras foram secas ao ar, passadas por peneiras com malha de $2 \mathrm{~mm}$, misturadas e analisadas quanto às suas propriedades químicas e físicas (Sampaio et al., 1995).

A mineralização de $\mathrm{N}$ foi medida segundo o método de Stanford \& Smith (1972), com modificações (Alves, 1989), principalmente para incluir a medida simultânea do C mineralizado (Salcedo et al., 1985). A cada amostra de $50 \mathrm{~g}$ foi adicionada quantidade de areia de quartzo, lavada com ácido cl orídrico e passada em peneira com malha de $1 \mathrm{~mm}$, calculada, para cada solo, de acordo com equação desenvolvida por Lueking \& Schepers (1986). As misturas foram postas em colunas de percolação, construídas com tubos de PVC, de $4 \mathrm{~cm}$ de diâmetro por $20 \mathrm{~cm}$ de altura, tendo na parte inferior tampa de PVC e na parte superior tampa de borracha com fechamento hermético. A tampa inferior tinha um pequeno tubo, com $1 \mathrm{~cm}$ de 
diâmetro, para permitir a saída do percolado, que também tinha tampa hermética de borracha. Na base interior inferior da coluna, foi colocada uma tela de náilon, com malha de $1 \mathrm{~mm}$, e, sobreela, uma camada de lã de vidro para evitar perda de solo. Da tampa superior pendia um recipiente de vidro, no espaço interno da coluna, entre ela e o solo. De cada amostra, foram preparadas três colunas e, ainda, como testemunha, três colunas com apenas $62 \mathrm{~g}$ de areia lavada.

Inicialmente, o $\mathrm{N}$ mineral $\left(\mathrm{N}-\mathrm{NH}_{4}+\mathrm{N}-\mathrm{NO}_{3}\right)$ das amostras foi percolado com $100 \mathrm{~mL}$ de solução de $\mathrm{CaCL}_{2} 0.01 \mathrm{~mol} \mathrm{~L}^{-1}$, em alíquotas de $20 \mathrm{~mL}$, seguida pela aplicação de duas alíquotas de $25 \mathrm{~mL}$ desol ução nutritiva destituída de $\mathrm{N}\left(\mathrm{CaSO}_{4}\right.$ 0,01 mol L-1, $\mathrm{MgSO}_{4}$ 0,002 mol L-1 e $\mathrm{KH}_{2} \mathrm{PO}_{4}$ 0,005 mol L-1). O excesso de solução foi removido por vácuo, $25 \mathrm{~mm}$ $\mathrm{Hg}$, aplicado ao tubo inferior da col una. No recipiente de vidro, foram colocados $10 \mathrm{~mL}$ de $\mathrm{NaOH} 1 \mathrm{~mol} \mathrm{~L}-1$, para absorver $\mathrm{OCO}_{2}$ desprendido na coluna, que foi fechada. As colunas foram mantidas em casa de vegetação, com temperatura variável durante o dia de 30 a $35^{\circ} \mathrm{C}$, durante todo o tempo de incubação que foi de 20 semanas, para as amostras de $0-20 \mathrm{~cm}$ de profundidade, e de 4 semanas, para as de $20-40 \mathrm{~cm}$. Neste tempo, as colunas foram abertas para retirada e reposição do $\mathrm{NaOH}$ e para percolação do N mineral, da forma supradescrita, em quatro interval os consecutivos de uma semana, seguidos, para as amostras de $0-20 \mathrm{~cm}$, de mais dois de duas semanas e três de quatro semanas.

Para determinação do $\mathrm{N}$ mineralizado, analisaramse, no percolado, oN-NH $\mathrm{NH}_{4}$ eoN-NO 3 por colorimetria (EPA, 1971). O C-CO ${ }_{2}$ absorvido pela solução de $\mathrm{NaOH}$ foi determinado titulando-se com $\mathrm{HCl}$ 0,05 mol L-1, potenciometricamente, no interval o de pH de 8,3 a 3,7 (Sampaio \& Salcedo, 1982).

O nitrogênio potencialmente mineralizável foi estimado com base nos valores de $\mathrm{N}$ mineralizado, acumulados no tempo, e três modelos de cinética, ajustando-se por regressão não-linear, pelo método terativoquase-Newton, no pacoteestatísticoStatistica (StatSoft, 1995). O primeiro modelo, exponencial simples (Stanford \& Smith, 1972), considera que existe apenas um reservatório de $\mathrm{N}$ mineralizável e é definido pela equação $\mathrm{Nt}=\mathrm{No}\left(1-\mathrm{e}^{-k t}\right)$, em que $\mathrm{Nt}=$ $\mathrm{N}$ mineralizado no tempo t; $\mathrm{No}=\mathrm{N}$ potencialmente mineral izável; e $\mathrm{k}=$ constante de mineral ização. $\mathrm{O}$ segundo modelo, exponencial duplo (Molina et al., 1980), considera que existem dois reservatórios de $\mathrm{N}$ potencial mente mineralizável, $\mathrm{No}_{1} \mathrm{eNo} \mathrm{O}_{2}$, com suas respectivas taxas de mineralização, $h$ e $k$, e é representado pela equação $\mathrm{Nt}=\mathrm{No}_{1}\left(1-e^{-h t}\right)+\mathrm{No}_{2}$ (1 - e-kt). O terceiro modelo, misto exponencial elinear (Seyfried \& Rao, 1988), considera um reservatório com mineralização, realizando-se de acordo com uma reação cinética de primeira ordem e outro infinito, correspondendo à mineralização constante no tempo e que seria a mineralização basal por um longo período, sendo representado pela equação $\mathrm{Nt}=\mathrm{No}_{1}$ ( 1 - e-kt) + ht. Outras equações foram ajustadas aos dados de $\mathrm{N}$ mineralizado, acumulados ou não (Ellert \& Bettany, 1988), mas não são apresentadas. As formas das curvas de mineralização foram ainda definidas pela equação de Richards (White \& Marinakis, 1991), representada por $\mathrm{dN}=(2(m+1) /$

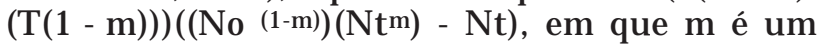
parâmetro que representa a forma da curva.

\section{RESULTADOS E DISCUSSÃO}

\section{Curvas de mineralização de $\mathbf{C}$ e de $\mathbf{N}$}

As quantidades líquidas de $\mathrm{N}$ orgânico mineralizado $\left(\mathrm{N}-\mathrm{NO}_{3}\right.$ e $\mathrm{N}-\mathrm{NH}_{4}$ ) foram muito variáveis (F iguras 1 e2). A pesar disto, as médias de $\mathrm{N}$ mineral acumulado foram significativamentemais altas na zona úmida que na semi-árida. Na camada de $0-20 \mathrm{~cm}$, nas 20 semanas deincubação, a diferença entreas zonas na produção de $\mathrm{N}$ mineral foi pequena (36 e $27 \mathrm{mg} \mathrm{kg}^{-1}$, nas zonas úmi da esemi-árida), mas, na camada de $20-40 \mathrm{~cm}$, nas 4 semanas da incubação, a diferença foi grande (25 e $7 \mathrm{mg} \mathrm{kg}^{-1}$, respectivamente). Comparando as mineralizações nas duas camadas, para o mesmo período de 4 semanas, as de $0-20 \mathrm{~cm}$ foram superiores às de 20 $40 \mathrm{~cm}$ na zona semi-árida, enquanto, na zona úmida, os valores atingiram mesma ordem de grandeza, chegando a ser maiores na camada subsuperficial em 4 dos 10 solos. Em geral, o potencial de mineral ização decresce com a profundidade do solo, como foi encontrado por Salcedo et al. (1985) em um solo da zona úmida de Pernambuco. O fato de a maioria dos sol os da zona úmi da não ter seguido este padrão geral pode estar ligado ao uso desses solos (9) com plantio de cana-de-açúcar, em contrastecom os da zona semi-árida (7) que foram amostrados sob vegetação nativa.

Tendências semelhantes às de $\mathrm{N}$, em termos de efeitos das zonas, ocorreram também nas curvas de mineralização de $\mathrm{C}\left(\mathrm{C}-\mathrm{CO}_{2}\right)$, mas o efeito da profundidade foi distinto na zona úmida (Figuras 3 e 4). As mineralizações foram superiores na zona úmida em relação à semi-árida e na camada superficial em relaçãoà subsuperficial (úmida, 0-20 e 20-40 cm, 446 e $177 \mathrm{mg} \mathrm{kg}^{-1}$; semi-árida, 0-20 e 2040, 368 e $66 \mathrm{mg} \mathrm{kg}^{-1}$ ). I sto indica que a matéria orgânica dos solos da zona úmida pode liberar maiores quantidades de $\mathrm{C}-\mathrm{CO}_{2}$ e fornecer quantidades maiores de $\mathrm{N}$ para as plantas que a da zona semi-árida, sobretudo considerando a faixa de 0-40 cm de profundidade. Entretanto, por causa das amplas faixas de variação e do número reduzido de solos, a diferença entre zonas não deve ser enfatizada.

Os dados da produção acumulada de $\mathrm{N}$ mineral, ao longo das 20 semanas de incubação, foram 


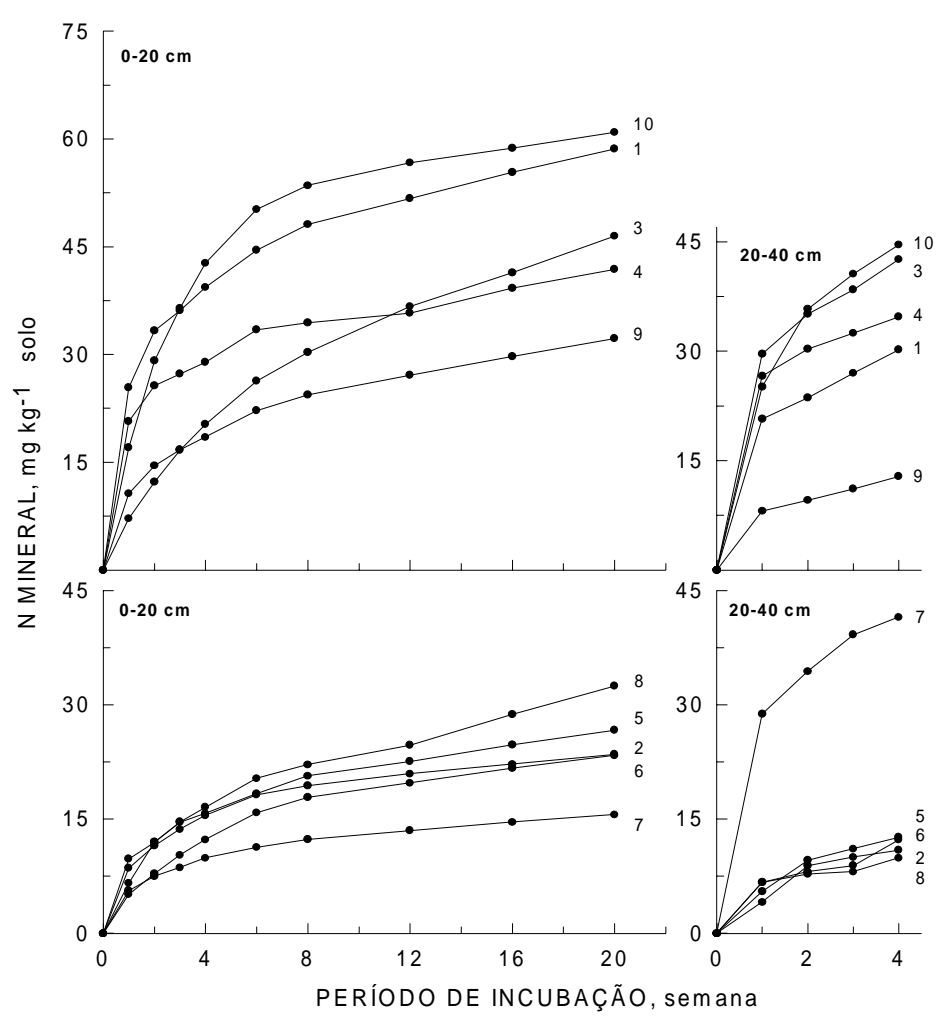

Figura 1. Mineralização acumulada de nitrogênio $\left(\mathrm{NH}_{4}{ }^{+}+\mathrm{NO}_{3}{ }^{-}\right)$em amostras das camadas de 0-20 e 20-40 cm de solos da zona úmida de Pernambuco, incubadas por 20 e 4 semanas, respectivamente.

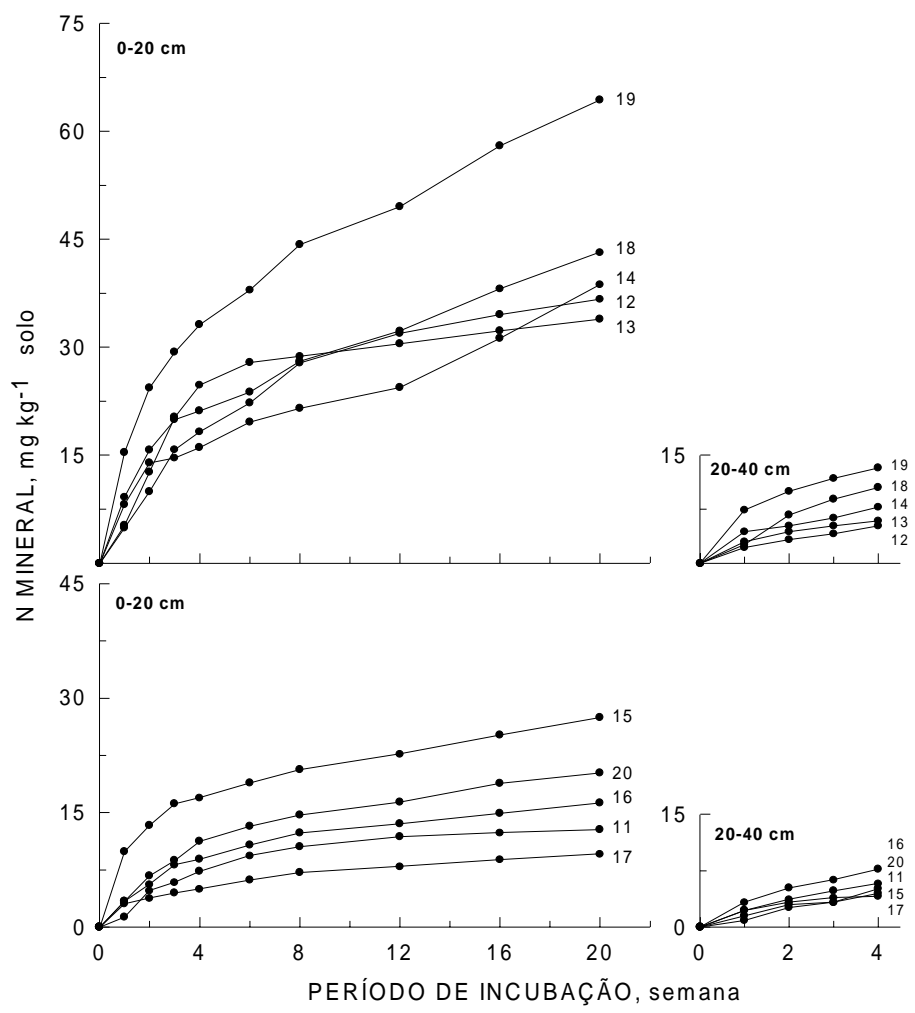

Figura 2. Mineralização acumulada de nitrogênio $\left(\mathrm{NH}_{4}{ }^{+}+\mathrm{NO}_{3}{ }^{-}\right)$em amostras das camadas de $0-20$ e 20-40 cm de solos da zona semi-árida de Pernambuco, incubadas por 20 e 4 semanas, respectivamente. 
ajustados pelos modelos exponencial simples, exponencial duplo emisto exponencial - linear (Ellert \& Bettany, 1988) e pela equação de Richards (White \& Marinakis, 1991). O ajustepelo model o exponencial simples (cinética de primeira ordem) levou a val ores de nitrogênio potencialmente mineralizável (No) muito próximos e, na maioria dos casos, inferiores aos valores mineralizados nas 20 semanas de incubação $\left(\mathrm{N}_{20}\right.$, Quadro 1). Valores inferiores seriam teoricamente impossíveis e até os pouco superiores parecem duvidosos porque pressupõem que todo ou quase todo o $\mathrm{N}$ mineralizável do solo teria sido mineralizado durante o período de incubação, restando muito pouco para qualquer ciclo seguinte de incubação ou cultivo. Val ores de No inferiores aos mineralizados foram obtidos para a maioria de 30 (Parentoni et al., 1988) e de 10 solos (Camargo et al., 1997) do Rio Grande do Sul e têm sido encontrados para sol os de clima temperado (Deans et al., 1986; Bonde \& Rosswall, 1987; Seyfried \& Rao, 1988). Os val ores da taxa de mineral ização variaram de 0,13 a 0,53 semana-1, confirmando que os solos podem ter taxas bem diferentes (J uma et al., 1984; Seyfried \& Rao, 1988).
$\mathrm{O}$ ajuste com dois reservatórios de $\mathrm{N}$ potencialmente mineralizável $\left(\mathrm{No}_{1}\right.$ e $\left.\mathrm{No}_{2}\right)$ diminuiu a raiz quadrada do quadrado médio residual (RQQMR, não mostrado) em 9 das 20 amostras, em relação ao ajuste com exponencial simples; em duas amostras, o RQQM R aumentou enas restantes foi semel hante nos dois tipos de ajuste. A literatura registra inúmeros casos de melhor ajuste ora com um reservatório ora com dois (Molina et al., 1980; J ones, 1984; Beauchamp et al., 1986; Deans et al., 1986; Bonde \& Rosswall, 1987; Dalal \& Mayer, 1987; Saito \& Ishii, 1987; Seyfried \& Rao, 1988; Nira \& Nishimune, 1993). Sete das amostras da zona semiárida e três da zona úmida tiveram tendência à linearização da mineralização (Figuras 1 e 2). Tendência semel hante foi encontrada por Seyfried \& Rao (1988), indicando não ser a cinética de primeira ordem com dois reservatórios adequada ao ajuste dos dados de alguns solos. Neste caso, os val ores de $\mathrm{No}_{2}$ foram muito altos (Quadro 1) e com interval os de confiança amplos (não mostrados). Nas amostras sem a tendência à linearização, os val ores de $\mathrm{No}_{1}+\mathrm{No}_{2}$ foram sempre iguais ou superiores aos de $\mathrm{N}$ mineralizado nas 20 semanas de incubação,

Quadro 1. Parâmetros das equações de ajuste dos dados de mineralização acumulada de N, durante 20 semanas de incubação $\left(\mathrm{N}_{20}\right)$, aos modelos exponencial simples, exponencial duplo, misto exponencial e linear e de Richards ( $R$ )

\begin{tabular}{|c|c|c|c|c|c|c|c|c|c|c|c|}
\hline \multirow{2}{*}{ Solo } & \multirow{2}{*}{$\mathbf{N}_{20}$} & \multicolumn{2}{|c|}{ Simples } & \multicolumn{4}{|c|}{ Duplo } & \multicolumn{3}{|c|}{ Misto } & \multirow{2}{*}{$\begin{array}{l}\mathbf{R} \\
\mathbf{m}\end{array}$} \\
\hline & & No & $\mathbf{k}$ & $\mathrm{No}_{\mathbf{1}}$ & $\mathrm{NO}_{2}$ & $\mathbf{k}$ & $\mathbf{h}$ & $\mathrm{No}_{1}$ & $\mathbf{k}$ & $\mathbf{h}$ & \\
\hline & \multicolumn{2}{|c|}{ — $\mathrm{mg} \mathrm{kg}^{-1} \_$} & semana $^{-1}$ & \multicolumn{2}{|c|}{ - $\mathrm{mg} \mathrm{kg}^{-1}-$} & \multicolumn{2}{|c|}{ — semana-1 _ } & $\mathrm{mg} \mathrm{kg}^{-1}$ & \multicolumn{2}{|c|}{ - semana ${ }^{-1}$ - } & \\
\hline & \multicolumn{11}{|c|}{ Zona úmida } \\
\hline 1 & 58,6 & 53,0 & 0,42 & 27,4 & 34,6 & 1,60 & 0,11 & 36,9 & 0,95 & 1,14 & $-4,53$ \\
\hline 2 & 23,5 & 21,9 & 0,34 & 7,4 & 16,1 & 1,61 & 0,17 & 16,1 & 0,57 & 0,38 & $-2,81$ \\
\hline 3 & 46,5 & 47,7 & 0,13 & 21,4 & $187^{(1)}$ & 0,31 & $0,007^{(1)}$ & 22,6 & 0,29 & 1,19 & $-0,11$ \\
\hline 4 & 41,9 & 37,1 & 0,53 & 22,1 & 22,2 & 1,84 & 0,10 & 27,1 & 1,21 & 0,77 & $-7,31$ \\
\hline 5 & 26,7 & 24,8 & 0,27 & 15,2 & 30,5 & 0,54 & 0,02 & 16,4 & 0,51 & 0,52 & 0,14 \\
\hline 6 & 23,4 & 22,8 & 0,20 & 16,0 & $68^{(1)}$ & 0,30 & $0,006^{(1)}$ & 16,2 & 0,30 & 0,36 & $-0,59$ \\
\hline 7 & 15,6 & 14,3 & 0,32 & 5,8 & 10,6 & 1,40 & 0,12 & 9,5 & 0,67 & 0,31 & $-3,46$ \\
\hline 8 & 32,5 & 29,7 & 0,21 & 10,5 & 34,6 & 1,26 & 0,05 & 14,1 & 0,76 & 0,92 & $-3,63$ \\
\hline 9 & 32,2 & 29,6 & 0,27 & 11,0 & 24,4 & 1,43 & 0,09 & 17,6 & 0,68 & 0,76 & $-3,21$ \\
\hline \multirow[t]{2}{*}{10} & 61,0 & 59,1 & 0,32 & 52,8 & $292^{(1)}$ & 0,38 & $0,001^{(1)}$ & 52,8 & 0,37 & 0,39 & 0,33 \\
\hline & \multicolumn{11}{|c|}{ Zona semi-árida } \\
\hline 11 & 12,8 & 13,0 & 0,20 & 6,5 & 6,5 & 0,20 & 0,20 & 13,9 & 0,19 & 0,00 & 1,32 \\
\hline 12 & 36,7 & 37,8 & 0,16 & 33,0 & $118^{(1)}$ & 0,18 & $0,002^{(1)}$ & 33,1 & 0,18 & 0,22 & 0,86 \\
\hline 13 & 33,9 & 33,0 & 0,29 & 31,8 & $81^{(1)}$ & 0,30 & $0,001^{(1)}$ & 31,8 & 0,30 & 0,07 & 2,43 \\
\hline 14 & 38,7 & 36,0 & 0,14 & 11,0 & $2.379^{(1)}$ & 1,19 & $0,000^{(1)}$ & 11,0 & 1,18 & 1,31 & $-1,93$ \\
\hline 15 & 27,4 & 24,4 & 0,34 & 14,2 & 31,2 & 0,96 & 0,03 & 15,3 & 0,85 & 0,61 & $-1,00$ \\
\hline 16 & 16,3 & 15,5 & 0,21 & 10,2 & $103^{(1)}$ & 0,36 & $0,003^{(1)}$ & 10,3 & 0,36 & 0,29 & 0,15 \\
\hline 17 & 9,6 & 9,0 & 0,22 & 2,2 & 8,2 & 5,24 & 0,11 & 4,8 & 0,63 & 0,25 & $-3,91$ \\
\hline 18 & 43,2 & 40,4 & 0,18 & 16,9 & $703^{(1)}$ & 0,71 & $0,002^{(1)}$ & 17,1 & 0,70 & 1,30 & $-0,63$ \\
\hline 19 & 64,4 & 60,1 & 0,20 & 26,5 & $153^{(1)}$ & 0,69 & $0,010^{(1)}$ & 28,6 & 0,63 & 1,80 & $-1,00$ \\
\hline 20 & 20,2 & 19,6 & 0,19 & 12,5 & $207^{(1)}$ & 0,32 & $0,002^{(1)}$ & 12,6 & 0,32 & 0,38 & 0,60 \\
\hline
\end{tabular}

(1) Parâmetro com valor variável para algumas combinações de $\mathrm{N}_{2}$ alto e $\mathrm{h}$ baixo, por ajuste de exponencial a segmento de curva quaselinear. 


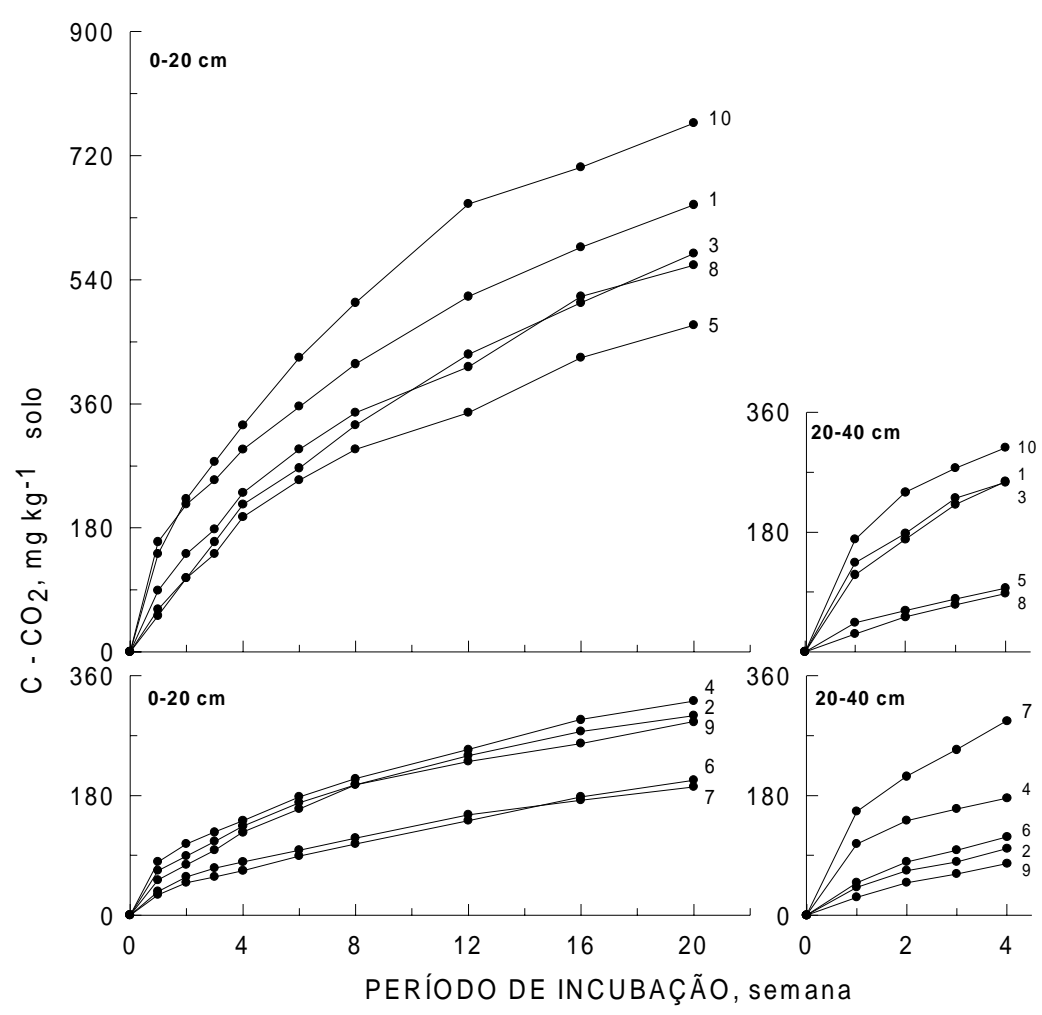

Figura 3. Mineralização acumulada de carbono $\left(\mathrm{C}-\mathrm{CO}_{2}\right)$ em amostras das camadas de $0-20$ e $20-40 \mathrm{~cm}$ de solos da zona úmida de Pernambuco, incubadas por 20 e 4 semanas, respectivamente.

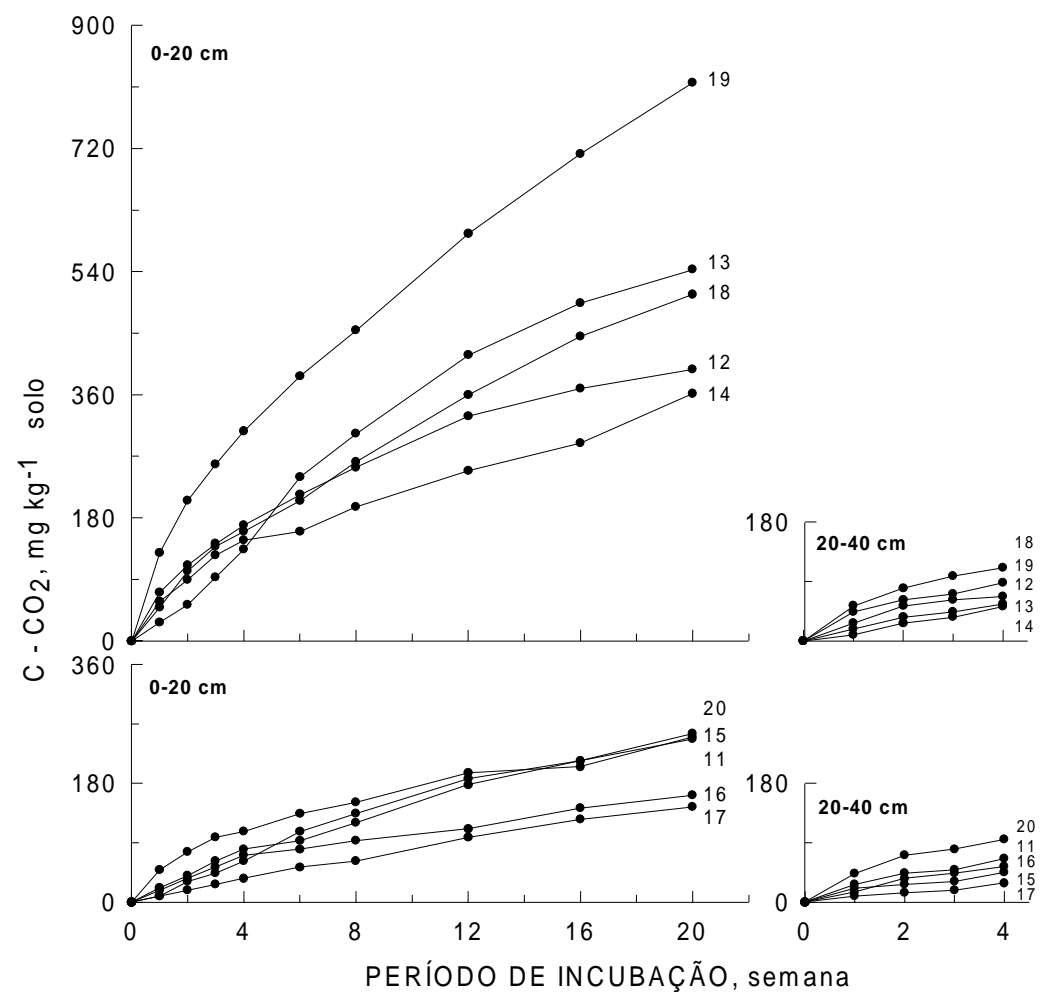

Figura 4. Mineralização acumulada de car bono $\left(\mathrm{C}-\mathrm{CO}_{2}\right)$ em amostras das camadas de 0-20 e $20-40 \mathrm{~cm}$ de solos da zona semi-árida de Pernambuco, incubadas por 20 e 4 semanas, respectivamente. 
evidenciando resultados mais coerentes que os do exponencial simples. Os potenciais de mineralização $\left(\mathrm{No}_{1}+\mathrm{No}_{2}\right)$ destes sol os sem tendência à linearização seriam equivalentes à faixa de 27 a $160 \mathrm{~kg} \mathrm{ha}^{-1}$ de $\mathrm{N}$, considerando-se a camada de $20 \mathrm{~cm}$ superficiais. Deste $\mathrm{N}$ potencialmente mineralizável, 20 a 50\% estariam no reservatório mais lábil $\left(\mathrm{No}_{1}\right)$, podendo ser mineralizados em menos de uma semana ( $\mathrm{k}$ próximo ou acima de 1 semana-1).

Para possibilitar o ajuste de curvas de mineralização com tendência à linearidade nos períodos finais de incubação, foi proposto o modelo misto de cinética de primeira ordem e dezero ordem (Seyfried \& Rao, 1988; Bonde et al., 1988). Por conta da partelinear, o model o não per mite o cál culo do N potencialmente mineralizável. Por outro lado, permiteo cálculo deuma taxa basal demineralização, passada a fase inicial de mineralização decrescente, com o esgotamento do $\mathrm{N}$ de fácil mineralização. $\mathrm{O}$ reservatório de $\mathrm{N}$ de fácil mineralização $\left(\mathrm{No}_{1}\right)$, em todos os casos (Quadro 1), foi menor que o $\mathrm{N}$ mineralizado experimental mente $\left(\mathrm{N}_{20}\right)$. As taxas basais de mineralização do modelo misto ( $h$, Quadro 1) dos 10 sol os com tendência à linearização variaram de 0,07 a 1,80 semana-1, uma faixa inferior à encontrada por Seyfried \& Rao (1988), de 0,73 a 5,15 semana-1, indicando que os sol os de Pernambuco teriam menor capacidade de fornecimento de $\mathrm{N}$ que os solos estudados por esses autores.

Outros tipos de desvio da cinética de primeira ordem têm sido observados além da linearização ao final da incubação. O principal deles é uma mineralização lenta no início da incubação, seguida do padrão da cinética de primeira ordem, como se houvesse um retardo no início da fase de mineralização rápida (Diaz-Fierros et al., 1988; Bonde \& Lindberg, 1988). Graficamente, corresponde a uma curva tendendoa sigmói de, como as dos únicos três solos cultivados da zona semi-árida (Figura 2, solos 11, 12 e 13). Tais desvios levaram à proposição do emprego da equação de Richards (White \& Marinakis, 1991), para identificar padrões de curvas. Os valores positivos de $\mathrm{m}$ indicam o retardo, mais acentuado com val ores mais al tos, como nos solos 13 e 11 (Quadro 1). Este retardo pode ser atribuído a uma população microbiana pequena ou pouco ativa, limitando a mineralização no início da incubação. Valores de $m$ próximos de zero indicam ajuste à cinética de primeira ordem com um único reservatório. Já valores negativos indicam mineralização muito rápida na fase inicial seguida de queda brusca, que se ajustam melhor com uma dupla exponencial ou com o model o misto. Foi o caso da mai oria dos sol os da zona úmida e al guns da zona semi-árida. Valores de $\mathrm{m}$ menores que -1 corresponderam a taxas de mineralização do reservatório lábil (k) da dupla exponencial maiores que 1, mostrandoter essereservatório mineralização muito rápida.
Não são mostrados os parâmetros de ajuste das curvas de mineral ização de $C$ que seguiram padrões semel hantes às de $\mathrm{N}$, apenas com maior tendência à linearização no final da incubação.

\section{C e $\mathbf{N}$ mineralizados e teores totais no solo}

A mineralização da matéria orgânica das amostras pode ser analisada com mais detalhe examinando-se a relação entre as quantidades de $C$ e de $\mathrm{N}$ mineralizados (Freytag $\&$ Rausch, 1984; Sal cedo et al., 1985). Elas foram bem correlacionadas tanto na camada superficial (Figura $5 a$ ) quanto na subsuperficial (Figura 6a). Na superficial, a relação C:N oscilou em torno de 12 , um val or um pouco mais altoque os encontrados por Freytag \& Rausch (1984) e por Sal cedo et al . (1985), mas inferior aos relatados por Araújo (1994). Na subsuperficial, a relação teve valores mais baixos que os da superficial, uma diferença explicada em parte pelo menor tempo de incubação. Isto porque, na camada de 0-20 cm, a relação foi maior nas últimas semanas de incubação, quando as curvas de $\mathrm{C}$ mineralizado tenderam à linearização e as de $\mathrm{N}$ a um decréscimo progressivo (F iguras 1 a 4). A relação mais baixa na fase inicial de mineralização, nas duas camadas, poderia ser explicada pel o consumo preferencial, nesta fase, de biomassa microbiana morta, um substrato relativamente rico em $\mathrm{N}$ (J enkinson \& Powlson, 1976). Na fase final da incubação, este substrato já estaria esgotado e a população microbiana estaria consumindo uma matéria orgânica menos lábil ecom maior relação $\mathrm{C}: \mathrm{N}$. Não houve diferenças significativas entre zonas quanto aos valores da relação.

As quantidades de $\mathrm{N}$ mineralizado, na camada de $0-20 \mathrm{~cm}$, foram proporcionais ao $\mathrm{N}$ total dos 20 sol os (F igura 5b), resultadotambém encontrado por outros autores (Campbell et al., 1981; Griffin \& Laine, 1983; Dalal \& Mayer, 1987; Camargo et al., 1997). Separando os solos por zona, os coeficientes de determinação entre as duas variáveis foram maiores (em ambas, $R^{2}=0,64 ; p<0,01$ ), o que está mais de acordo com o fato de o $\mathrm{N}$ total mineralizado nos solos da zona úmida (média de 5,9\%) ter sido significativamente superior ao da zona semi-árida (3,9\%). A maior proporção do $\mathrm{N}$ total mineralizado nos sol os da zona úmida éo componentequefez com que as quantidades absolutas mineralizadas nesta zona fossem superiores às da zona semi-árida (tratadas na seção anterior), já que os teores de $\mathrm{N}$ total, em média, foram menores na zona úmida (610 mg kg-1) que na semi-árida (770 mg kg-1). No trabalho de Parentoni et al. (1988), amostras superficiais de solos do Rio Grande do Sul mineralizaram menos, em geral, de seu $\mathrm{N}$ total $(0,7$ a $2,4 \%)$, em 30 semanas, que os de ambas as zonas de Pernambuco, mas no trabalho de Camargo et al . (1997), também com sol os do Rio Grande do Sul, em 32 semanas, as proporções do $\mathrm{N}$ total mineralizado $(5,9$ a $13,9 \%)$ foram semelhantes às dos solos de 
Pernambuco. Fora do Brasil, têm sido encontradas faixas semelhantes (Saito \& I shii, 1987; Bonde et al., 1988) e até mais altas (Campbell et al., 1981; Dalal \& Mayer, 1987).

Na camada de $20-40 \mathrm{~cm}$ de profundidade, a relação entre $\mathrm{N}$ mineralizado e $\mathrm{N}$ total (não mostrada) não foi significativa para os 20 solos, em conjunto, ou para os solos separados por zona, e as proporções também foram mais altas na zona úmida $(3,1$ a $14,9 \%$, média $7,1 \%)$ que na semi-árida $(0,6$ a $3,9 \%$, média $2,1 \%$ ), sem que houvesse diferença significativa no $\mathrm{N}$ total (350 e $390 \mathrm{mg} \mathrm{kg}^{-1}$ ). A falta de proporcionalidade entre $\mathrm{N}$ mineralizado e $\mathrm{N}$ total indica que, nesta camada, a qualidade da matéria orgânica dos sol os é distinta, ao contrário da camada superficial, que parece mais similar em qualidade. A diferença entrezonas mostra uma mai or labilidade dos compostos nitrogenados da matéria orgânica formada na zona úmida.

As quantidades de $\mathrm{C}$ mineralizado foram relacionadas positivamente com as de $C$ total, em ambas as camadas (F iguras 5 ce $6 b$ ), eas proporções, como as de $\mathrm{N}$ mineralizado, foram maiores na zona úmida que na semi-árida (úmida, 0-20 e 20-40 cm, 5,6 e 2,0\%; semi-árida 4,9 e 1,5\%). Parte dessas diferenças pode ser explicada pelos maiores teores de $C$ total nos sol os da zona úmida (0-20 e $20-40 \mathrm{~cm}$, 8,9e8,8 $\left.\mathrm{g} \mathrm{kg}^{-1}\right)$ quenos da semi-árida (7,6 e 4,9 g kg-1), principalmente na camada de $20-40 \mathrm{~cm}$. Na zona úmida, nesta camada, os teores médios de $C$ total foram quase iguais nas duas profundidades. $\mathrm{Na}$ maioria dos solos, o mais usual é que o teor de $\mathrm{C}$ total decresça com a profundidade (Volkoff \& Cerri, 1988), o quetambém foi encontrado em sol o cul tivado com cana, na zona úmida de Pernambuco (Sal cedo et al., 1985). Não há uma explicação clara para esta semel hança nas duas camadas da zona úmida, mas vale lembrar que el a também ocorreu em relação ao $\mathrm{N}$ mineralizado, como foi discutido anteriormente.

Outra forma de analisar as relações de mineral izaçãoé considerar a relação( $C$ mineralizado/C total)/(N mineralizado/N total) que dá uma idéia da disponibilidade relativa do $\mathrm{C}$ e do $\mathrm{N}$ da matéria orgânica para mineralização. Um valor próximo de 1 significa que os dois elementos têm a mesma disponibilidade para os microrganismos do solo. Val ores mais al tos que 1 indicam que o estoque de $C$ está sendo mineralizado mais rapidamente que o de $\mathrm{N}$. É o caso de sol os com a adição recente de compostos pobres em $\mathrm{N}$ e defácil decomposição, como pal has. Em geral, as adições têm pouco efeito sobre os teores totais, mas as mineralizações podem aumentar muito e de forma não-proporcional. Os valores também podem ser maiores que 1 , se o $\mathrm{N}$ mineralizado estiver sendo perdido do sistema, que, nestetrabal ho, teria deser deforma gasosa. Valores menores que 1 indicam maior disponibilidade relativa do N, ou seja, uso de substratos ricos em N, como a biomassa microbiana do solo.
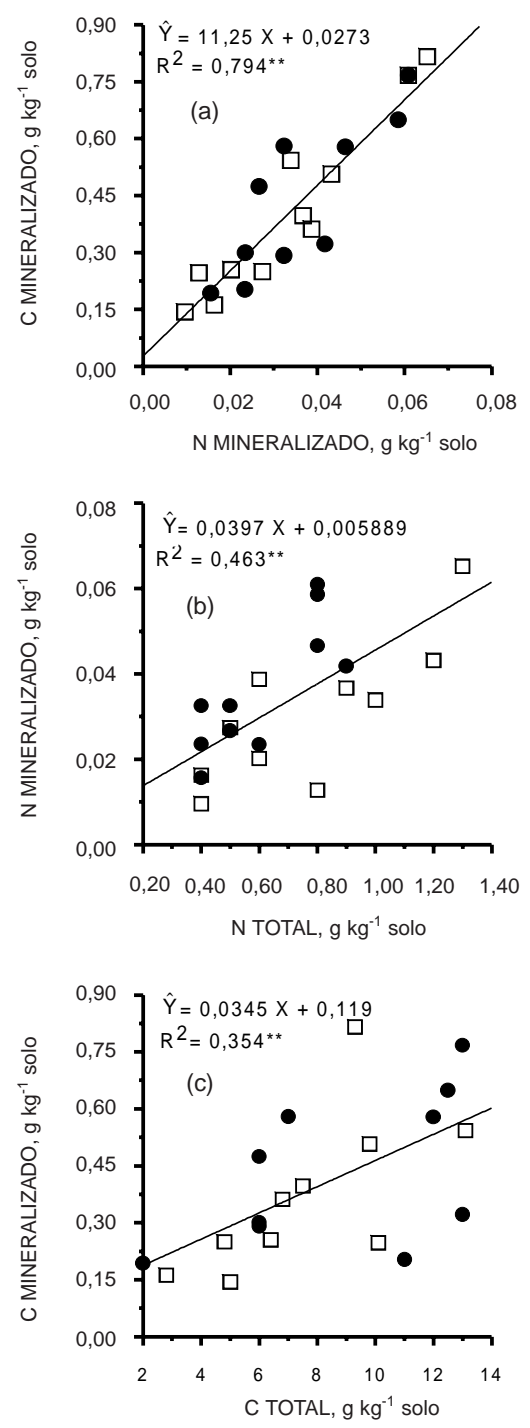

Figura 5. Relação entre os valores de $\mathbf{C}$ e de $\mathbf{N}$ mi neralizados em 20 semanas de incubação (a), de $\mathbf{N}$ mineralizado e de $\mathbf{N}$ total (b) e de $\mathbf{C}$ mineralizado e de C total (c), em amostras das camadas de $0-20 \mathrm{~cm}$ de solos da zona úmida (círculos) e da zona semi-árida (quadrados) de Pernambuco.

Na camada superficial da maioria dos solos (8) da zona semi-árida, os valores foram superiores a 1 (Quadro 2), indicando mineralização de substrato relativamente pobre em $\mathrm{N}$. E m sete dos sol os da zona úmida ocorreu o oposto, indicando que a proporção do $\mathrm{C}$ total que mineralizou foi menor que a do $\mathrm{N}$ total. O mesmo aconteceu com quase todos os sol os (19) das duas zonas, na camada de $20-40 \mathrm{~cm}$, tendo também os da zona úmi da val ores mais altos que os da semi-árida. Os valores baixos na camada de 20$40 \mathrm{~cm}$ podem ser atribuídos ao tempo curto de incubação, quando prevalecia a mineralização de biomassa morta, um substrato rico em N. As diferenças entrezonas, nas duas camadas, são mais difíceis de explicar. 

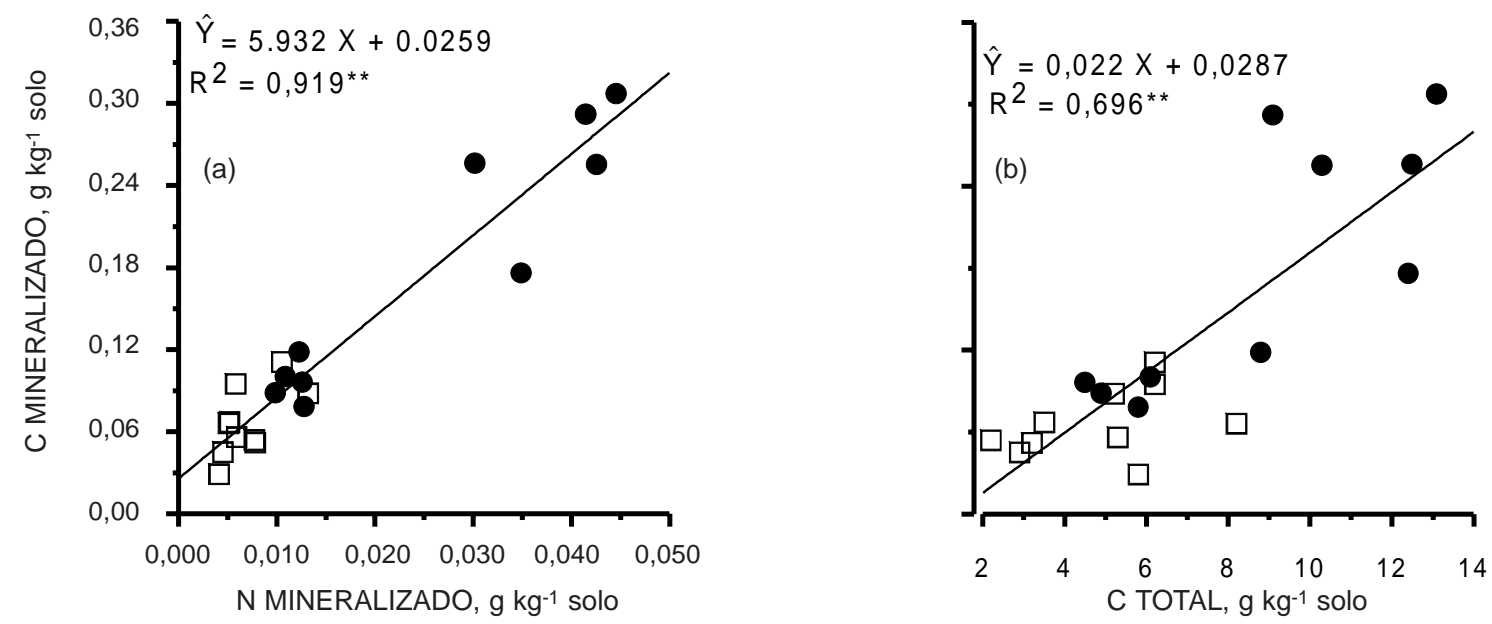

Figura 6. Relação entre os valores de $\mathbf{C}$ e de $\mathbf{N}$ mineralizados em 4 semanas de incubação (a) e de C mineralizado e de C total (b), em amostras das camadas de 20-40 cm de solos da zona úmi da (círculos) e da zona semi-árida (quadrados) de Pernambuco.

Quadro 2. Relações entre as proporções de C e N totais (Ct e Nt) mineralizados (Cm e Nm), em 20 e em 4 semanas de incubação, em amostras das camadas de 0-20 e20-40 cm de profundidade, respectivamente, de solos da zona úmida e da semi-árida, entre o $\mathrm{N}$ mineralizado $(\mathrm{Nm})$ acrescido do $\mathbf{N}$ mineral inicial (Ni) e o $\mathbf{N}$ absorvido por plantas cultivadas durante 30 dias ( $\mathrm{Na1}$ ) e entre o $\mathrm{N}$ mineralizado de 4 a 8 semanas de incubação ( $\mathbf{N m 8}$ ) e o $\mathbf{N}$ absorvido pelas plantas em um segundo cultivo por $\mathbf{3 0}$ dias (Na2)

\begin{tabular}{|c|c|c|c|c|c|}
\hline \multirow{2}{*}{ Solo } & \multicolumn{3}{|c|}{$0-20 \mathrm{~cm}$} & \multicolumn{2}{|c|}{$20-40 \mathrm{~cm}$} \\
\hline & $(\mathrm{Cm} / \mathrm{Ct}) /(\mathrm{Nm} / \mathrm{Nt})$ & $(\mathrm{Nm} 4+\mathrm{Ni}) / \mathrm{Na}_{1}$ & $\mathrm{Nm} 8 / \mathrm{Na}_{2}$ & $(\mathrm{Cm} / \mathrm{Ct}) /(\mathrm{Nm} / \mathrm{Nt})$ & $(\mathrm{Nm4}+\mathrm{Ni}) / \mathrm{Na}_{3}$ \\
\hline & \multicolumn{5}{|c|}{ Zona úmida } \\
\hline 1 & 0,71 & 1,37 & 0,76 & 0,34 & 1,14 \\
\hline 2 & 0,85 & 1,63 & 0,68 & 0,30 & 3,78 \\
\hline 3 & 0,83 & 1,54 & 1,29 & 0,23 & 1,98 \\
\hline 4 & 0,53 & 1,66 & 0,59 & 0,20 & 1,09 \\
\hline 5 & 1,47 & 1,88 & 1,16 & 0,51 & 1,10 \\
\hline 6 & 0,44 & 1,31 & 0,93 & 0,44 & 0,76 \\
\hline 7 & 2,27 & 1,56 & 0,46 & 0,31 & 1,03 \\
\hline 8 & 1,28 & 2,09 & 0,79 & 0,54 & 1,59 \\
\hline 9 & 0,68 & 1,49 & 0,82 & 0,21 & 0,90 \\
\hline 10 & 0,78 & 1,96 & 1,67 & 0,16 & 1,19 \\
\hline \multirow[t]{2}{*}{ Média } & 0,79 & 1,65 & 0,92 & 0,28 & 1,46 \\
\hline & \multicolumn{5}{|c|}{ Zona semi-árida } \\
\hline 11 & 1,54 & 0,75 & 0,60 & 0,94 & 0,52 \\
\hline 12 & 1,30 & 1,13 & 1,56 & 1,47 & 0,54 \\
\hline 13 & 1,22 & 1,08 & 0,34 & 0,71 & 0,63 \\
\hline 14 & 0,83 & 1,00 & 0,61 & 0,42 & 0,86 \\
\hline 15 & 0,95 & 1,23 & 0,54 & 0,69 & 0,93 \\
\hline 16 & 1,43 & 1,48 & 0,70 & 0,96 & 0,85 \\
\hline 17 & 1,20 & 0,48 & 0,63 & 0,85 & 0,27 \\
\hline 18 & 1,43 & 0,88 & 0,82 & 0,68 & 0,75 \\
\hline 19 & 1,75 & 1,14 & 0,79 & 0,51 & 0,89 \\
\hline 20 & 1,19 & 1,30 & 0,76 & 0,79 & 0,53 \\
\hline M édia & 1,23 & 1,05 & 0,74 & 0,72 & 0,68 \\
\hline
\end{tabular}


Os solos da zona úmida apresentaram uma matéria orgânica mais lábil que os da zona semiárida, tantoem relaçãoa $\mathrm{N}$ quanto ao C, eos substratos mineralizados foram relativamente mais ricos em $\mathrm{N}$. Apesar da grande variabilidade entre sol os, os da zona úmida tenderam a ter mai ores teores de $C$ total que os da zona semi-árida, principalmente na camada subsuperficial, mas não maiores teores de $\mathrm{N}$ total. Assim, parece que o $\mathrm{N}$ total da zona úmida teria uma taxa de renovação maior que a da semiárida. $\mathrm{O}$ maior teor de $\mathrm{C}$ total e a maior labilidade de $\mathrm{C}$ e de $\mathrm{N}$ podem resultar de um aporte maior $\mathrm{e}$ mais constante de material orgânico ao solo, na zona úmida, formando uma matéria orgânica com menor tempo de resi dência. O mai or aporte na zona úmida pode decorrer deseu maior potencial de produtividade primária. As relações de mineralização na camada de $20-40 \mathrm{~cm}$ de profundidade foram menos claras que na camada superficial, incluindo a falta de proporcionalidade entre $\mathrm{N}$ mineralizado e $\mathrm{N}$ total. É possível que a renovação da matéria orgânica na camada subsuperficial seja mais complexa, sendo os aportes mais dependentes do sistema radicular que da transferência de material caído na superfície do solo. Sabe-se muito pouco sobre aportes do sistema radicular em sol os do nordeste e do Brasil, em geral.

\section{Mineralização e absorção de $\mathbf{N}$ por plantas}

As quantidades de $\mathrm{N}$ mineralizado na camada de $0-20 \mathrm{~cm}$ foram bem correlacionadas com as de $\mathrm{N}$ absorvido por plantas de sorgo, cultivadas em casa de vegetação com amostras desses mesmos solos (Sampaio et al., 1995), tanto comparando-se o N mineralizado em 4 semanas com o $\mathrm{N}$ absorvido no cultivo por 30 dias $(r=0,62 ; p<0,01)$ quanto o $\mathrm{N}$ mineralizado em 8 semanas com o $\mathrm{N}$ absorvido nos dois cultivos sucessivos de 30 dias cada ( $r=0,67$; $p<0,01$ ). Camargo et al. (1997) também encontraram boa correlação entre mineralização e absorção de $\mathrm{N}$ por plantas cultivadas em vasos.

$\mathrm{O}$ fato de o $\mathrm{N}$ mineral lixiviado e o do absorvido pelo sorgo originarem-se do mesmo processo de liberação do $\mathrm{N}$ orgânico do solo justificaria não só correlações positivas mas também semelhanças entre os valores das duas medidas. Para esta comparação, foi necessário acrescentar ao N mineralizado o $\mathrm{N}$ mineral inicialmentepresentenas amostras (Ni), uma vez que, no ensaio em vasos, este $\mathrm{N}$ é aproveitado pelas plantas, enquanto, no experimento de incubação, ele não é computado (lixiviação no tempo zero). A razão $(\mathrm{Nm} 4+\mathrm{Ni}) / \mathrm{Na}_{1}$ (Quadro 1), para as 4 semanas de incubação e 30 dias de crescimento do sorgo, foi maior que 1 em todos os solos, exceto três do semi-árido, e a média da zona úmida $(1,65)$ foi significativamente maior que a do semi-árido (1,05). I sso indica que, em geral, há uma eficiência maior quanto à retirada do $\mathrm{N}$ do solo por lixiviação que pelas plantas, sobretudo na zona úmida.
J á o $\mathrm{N}$ mineral lixiviado de 4 a 8 semanas, nas mesmas amostras superficiais, foi menor que a absorção do segundo período de cultivo em 7 dos 10 sol os da zona úmida e em 9 dos 10 sol os da zona semi-árida (Quadro 1, Nm8/ $\mathrm{Na}_{2}$ ). Provavelmente contribuiu para esta diferença o fato de a mineralização ter sido medida sem nenhuma interrupção, enquanto o segundo cultivo foi precedido da retirada do solo dos vasos (separação deraízes, secagem, peneiramento, enchimento). Esse manuseio, em geral, causa morte da biomassa microbiana e liberação de $\mathrm{N}$ mineral ( enkinson \& Powlson, 1976; Ross et al., 1985). J untando-seos dois períodos (0-4 + 4-8 semanas de incubação e $30+30$ dias de cultivo), predomina o efeito do período inicial, por seus val ores absolutos mais altos.

Nas amostras de $20-40 \mathrm{~cm}$ de profundidade, o N mineralizado nas quatro semanas de incubação também foi correlacionado ( $r=0,67 ; p<0,01)$ com oN absorvido em 30 dias de cultivo (Sampaio et al., 1995) etodos os valores da relação ( $\mathrm{Nm} 4+\mathrm{Ni}$ )/Na $/ \mathrm{Na}_{1}$ nos sol os da zona úmi da foram mai ores que 1 , exceto os de dois sol os, enquanto todos os da zona semi-árida ficaram abaixo de 1 (Quadro 2). É inter essante registrar que o val or extremamente mais baixo na zona semi-árida correspondeu ao solo que teve o mesmo comportamento na camada superficial (solo 17).

A maior eficiência da lixiviação pode estar relacionada com a retirada total, com a lixiviação semanal, do N mineral acumulado, em comparação com uma retirada baixa pelas plantas no seu período inicial de crescimento, quando as raízes não exploram todo o vol ume de solo do vaso. Os valores mais baixos que 1 , principal mente os muito baixos, de alguns solos da zona semi-árida sugerem uma limitação do processo de mineralização, com as lixiviações contínuas, ou uma intensificação da mineralização, com a presença de plantas. Não há uma explicação clara para a maior eficiência da lixiviação nos sol os da zona úmida que nos da semiárida, considerando que, em ambas as zonas, os sol os têm ampla variação de características físicas e químicas. A diferença mais notável é a já citada: os solos da zona úmida serem predominantemente cultivados e os da zona semi-árida estarem sob vegetação nativa.

\section{CONCLUSÕES}

1. Os solos apresentaram distintos padrões de mineral ização aol ongo da incubação. Alguns tiveram a fase inicial com retardo e outros com grande fluxo e, na fase final, houve estabilização ou queda progressiva. Os valores de $\mathrm{N}$ potencialmente mineralizável calculados pelo modelo exponencial simples foram, na maioria dos casos, inferiores aos efetivamente mineralizados nas 20 semanas de incubação, o que seria teoricamente impossível. O 
model o com duas exponenciais deu resultados mais realistas. As curvas de $\mathrm{C}$ tenderam mais à estabilização final que as de $\mathrm{N}$.

2. As quantidades de $\mathrm{C}$ e de $\mathrm{N}$ mineralizados durante a incubação foram significativamente relacionadas entresi e com os teores de $\mathrm{C}$ e $\mathrm{N}$ totais do solo. As quantidades de $\mathrm{N}$ mineralizado foram também significativamente correlacionadas com as quantidades de $\mathrm{N}$ absorvidas por plantas, em ensaio em potes, anteriormente realizado, com os mesmos solos.

3. Os solos da zona úmida tiveram uma matéria orgânica mais lábil que os da zona semi-árida, tanto em relação a $\mathrm{N}$ quanto ao $\mathrm{C}$, e os substratos mineralizados foram relativamente mais ricos em $\mathrm{N}$. Apesar da grande variabilidade entre sol os, os da zona úmi da tenderam a ter mai ores teores de $C$ total e maior mineralização de $C$ que os da zona semiárida, principalmentena camada subsuperficial, mas não maiores teores de $\mathrm{N}$ total. Assim, parece que o $\mathrm{N}$ total da zona úmida teria uma taxa de renovação maior que a da semi-árida.

\section{LITERATURA CITADA}

ALVES, G.D. Mineralização de nitrogênio e de carbono em vinte solos de Pernambuco e absorção de nitrogênio pelo sorgo (Sorghum bicol or L. Moench.). Recife, Universidade Federal Rural de Pernambuco, 1989. (Tese de Mestrado)

ARAÚJ O, A.M.S. Mineralização do $\mathrm{C}$ e do $\mathrm{N}$ em solo cultivado com cana-de-açúcar, com e sem fertilização nitrogenada. Recife, Universidade Federal Rural de Pernambuco, 1994. (Tese de Mestrado).

BEAUCHAMP, E.G.; REYNOLDS, W.D.; BRASCHEVILLENEUVE, D. \& KIRBY, K. Nitrogen mineralization kinetics with different soil pretreatments and cropping histories. Soil Sci. Soc. Am. J ., 50:1478-1483, 1986.

BONDE, T.A. \& LINDBERG, T. Nitrogen mineralization kinetics in soil during long-term aerobic laboratory incubations: a case study. J . Environ. Qual., 17:414-417, 1988.

BONDE, T.A. \& ROSSWALL, T. Seasonal variation of potentially mineralizable nitrogen in four cropping systems. Soil Sci. Soc. Am. J ., 51:1508-1514, 1987.

BONDE, T.A.; SCHNÜRER, J . \& ROSSWALL, T. Microbial biomass as a fraction of potentially mineralizable nitrogen in soils from long-term field experiments. Soil Biol. Biochem., 20:447-452, 1988.

CAMARGO, F.A.O; GIANELLO, C. \& VIDOR, C. Potencial de mineralização do nitrogênio em sol os do Rio Grande do Sul. R. Bras. Ci. Solo, 21:575-579, 1997.

CAMPBELL, C.A.; MYERS, R.J.K. \& WEIER, K.L. Potentially mineralizable nitrogen, decomposition rates and their relationship to temperature for five Queensland soils. Aust. J . Soil Res., 19:323-332, 1981.
DALAL, R.C. \& MAYER, R.J . Long-term trends in fertility of soils under continuous cultivation and cereal cropping in Southern Queensland. VII. Dynamics of nitrogen mineralization potentials and microbial biomass. Aust. J . Soil Res., 25:461-472, 1987.

DEANS, J.R.; MOLINA, J .A.E. \& CLAPP, C.E. Models for predicting potentially mineralizable nitrogen and decomposition rate constants. Soil Sci. Soc. Am. J ., 50:323$326,1986$.

DIAZ-FIERROS, F.; VILLAR, M.C.; GIL, F.; CARBALLAS, M.; LEIROS, M.C.; CARBALLAS, T. \& CABANEIRO, A. Effect of cattle slurry fractions on nitrogen mineralization in soil. J . Agric. Sci., 110:491-497, 1988.

DÖBEREINER, J . O nitrogênio na agricultura brasileira. In: SIMPÓSIO BRASILEIRO SOBRE NITROGÊNIO EM PLANTAS, 1., I taguaí, 1990. Anais. Itaguaí, Sociedade Brasileira de Fisiologia Vegetal, 1990. p.3-26.

ELLERT, B.H. \& BETTANY, J R. Comparison of kinetic models for describing net sulfur and nitrogen mineralization. Soil Sci. Soc. Am. J ., 52:1692-1702, 1988.

EPA - ENVIRONMENTAL PROTECTION AGENCY. Methods for chemical analysis of waters and wastes. Cincinnati, EPA, 1971. 312p.

FREYTAG, H.E. \& RAUSCH, H. Beziehung zwischen der C- und der N-Mineral isierung der organischen Bodensubstanz. Zbl. Mikrobiol., 139:537-544, 1984.

GRIFFIN, G.F . \& LAINE, A.F. Nitrogen mineralization in soils previously amended wtih organic wastes. Agron. J ., 75:124129, 1983.

JENKINSON, D.S. \& POWLSON, D.S. The effects of biocidal treatments on metabolism in soil. IV The decomposition of fumigated organisms in soil. Soil Biol. Biochem., 8:203-208, 1976.

J ONES, C.A. Estimation of an active fraction of soil nitrogen. Comm. Soil Sci. Plant Anal., 15:23-32, 1984.

J UMA, N.G.; PAUL, E.A. \& MARY, B. Kinetics analysis of net nitrogen mineralization in soil. Soil Sci. Soc. Am. J ., 48:753$757,1984$.

KINJ O, T.; MARCOS, Z.Z. \& J ACOB, O.M. Produção de nitrato por incubação de amostras do horizonte Ap de solos da região canavieira de Piracicaba. R. Bras. Ci. Solo, 2:103106, 1978.

LEMOS, E.E.P; SALCEDO, I.H. \& SAMPAIO, E.V.S.B. Comparação entre o $\mathrm{N}$ mineralizado através de incubações com e sem percolação e o $\mathrm{N}$ absorvido pel o milheto em solo podzólico vermelho- amarelo. R. Bras. Ci. Solo, 12:127-130, 1988.

LUEKING, M.A. \& SCHEPERS, J .S. Achieving desired moisture conditions in potentially mineralizable incubation studies. Soil Sci. Soc. Am. J ., 50:1370-1373, 1986.

MALAVOLTA, E. Pesquisa com nitrogênio no Brasil - passado, presente e perspectivas. In: SIMPOSIO BRASILEIRO SOBRE NITROGÊNIO EM PLANTAS, 1., I taguaí, 1990. Anais. I taguaí, Sociedade Brasileira de Fisiologia Vegetal, 1990. p.89-177. 
MOLINA, J .A.E.; CLAPP, C.E. \& LARSON, W.E. Potentially mineralizablenitrogen in soil: thesimple exponential model does not apply for the first 12 weeks of incubation. Soil Sci. Soc. Am. J ., 44:442-443, 1980.

NIRA, R. \& NISHIMUNE, A. Studies on nitrogen mineralization properties of Tokachi soils by kinetic analysis. Soil Sci. Plant Nutr., 39:321-329, 1993.

PARENTONI, S.N.; FRANÇA, G.E. \& BAHIA FILHO, A.F.C. Avaliação dos conceitos de quantidade e intensidade de mineral ização de nitrogênio para trinta sol os do Rio Grande do Sul. R. Bras. Ci. Solo, 12:225-229, I988.

POTTKER, D. \& TEDESCO, M.J . Efeito do tipo e tempo de incubação sobre a mineralização da matéria orgânica e nitrogênio total em solos do Rio Grande do Sul. R. Bras. Ci. Solo, 3:20-24, 1979.

ROSS, D.J .; SPEIR, T.W.; TATE, K.R. \& ORCHARD, V.A. Effects of sieving on estimations of microbial biomass, and carbon, and nitrogen mineralization, in soil under pasture. Aust. J . Soil Res, 23:319-324, 1985.

SAITO, M. \& ISHII, K. Estimation of nitrogen mineralization in corn-grown fields based on mineralization parameters. Soil Sci. Plant Nutr., 33, 555-566, 1987.
SALCEDO, I.H.; SAMPAIO, E.V.S.B. \& ALVES, G.D. Mineralização do carbono e do nitrogênio em solo cultivado com cana-de-açúcar. R. Bras. Ci. Solo, 9:33-38, 1985.

SAMPAIO, E.V.S.B. \& SALCEDO, I.H. Decomposição de pal ha de milho marcada e incorporação do ${ }^{14} \mathrm{C}$ à biomassa microbiana de um latossolo vermel ho - amarelo. R. Bras. Ci. Solo, 6:29-32, 1982.

SAMPAIO, E.V.S.B.;SALCEDO, I.H.;SILVA, V.M. \& ALVES, G.D. Capacidade de suprimento de $\mathrm{N}$ e resposta à fertilização de 20 sol os de Pernambuco. R. Bras. Ci. Solo, 20:269-279, 1995.

SEYFRIED, M.S. \& RAO, P.S.C. Kinetics of nitrogen mineralization in Costa Rican soils: model evaluation and pretreatment effects. Plant Soil, 106:159-169, 1988.

STANFORD, G. \& SMITH, S.J. Nitrogen availability mineralization potentials of soils. Soil Sci. Soc. Am. Proc., 36:465-472, 1972.

STATSOFT, G. Inc. Statistica for Windows (computer program manual). Tulsa, 1995

VOLKOFF, B \& CERRI, C.C. L'humus des sols du Brésil. Nature et relations avec l'environnement. Cah. ORSTOM, 24:8395, 1988.

WHITE, C.S. \& MARINAKIS, Y.D. A flexible model for quantitative comparisons of nitrogen mineralization patterns. Biol. Fertil. Soils, 11:239-244, 1991. 